\title{
Die Sedimente in der Höhlenruine von Hunas (Nördliche Frankenalb)
}

\author{
(Vorläufiger Bericht) ${ }^{1}$ ) \\ Von Karl Brunnacker, München \\ Mit 1 Abbildung im Text
}

\begin{abstract}
Wie früher dargelegt (K. BrunNaCKer 1956), sollen durch die Untersuchung von Höhlensedimenten in erster Linie folgende Probleme einer Klärung näher gebracht werden: Die Unterscheidung von warm- und kaltklimatischen Sedimenten (z. B. Sinter-Frostschutt) steht im Vordergrund der gesamten Untersuchungen. Hierbei kann auf den Grundvorstellungen von R. LAIS (1941) aufgebaut werden. Doch ist dabei weiter von Bedeutung, ob es sich um dem (infolge Abwitterung laufend zurïckweichenden) Eingang nahe Ablagerungen handelt, oder ob Material des Höhleninneren vorliegt. Ferner ist zu klären, ob autochthones oder infolge Einwehung bzw. Einschwemmung allochthones Material den Gesteinskörper aufbaut oder daran beteiligt ist. Schließlich sind nachträgliche Veränderungen im Sediment infolge pedogener Vorgänge einschließlich der spezifischen Art der Gesteinsverwitterung und ferner die durch evtl. auch nur kurzstreckige Umlagerung am Höhlenboden bedingten Schichtlücken bzw. -verdoppelungen zu erfassen. Diese Fragen allein durch eine laboratoriumsmäßige Auswertung der Sedimentproben klären zu wollen ist nicht sinnvoll; es kommt vielmehr auf eine Verknüpfung der hierbei gemachten Befunde mit den am Aufschluß möglichen Beobachtungen an.
\end{abstract}

Die Sedimente in der Höhle von Hunas (ca. $35 \mathrm{~km}$ östlich von Nürnberg) wurden seit 1957 auf Anregung von Herrn Prof. Dr. Fl. Heller - dem Entdecker und Ausgräber der Höhle - von mir untersucht (vgl. Heller 1963). Gemäß dem bisherigen Stand dieser Arbeiten lassen sich folgende zusammenfassende Angaben machen:

Die Höhle ist im Frankendolomit angelegt; deshalb sei zuerst auf den eigenartigen Verwitterungsablauf des Dolomitgesteins, der sich scheinbar anders verhält als bei Kalkstein, hingewiesen. Im Rahmen chemischer, jedoch nicht unbedingt warmklimatischer Lösungsvorgänge wird nämlich dem Dolomit ein leichtlöslicher Bestandteil entzogen. Damit „vermorscht" das Gestein zusehends und zerfällt schließlich zur feinsandigen „Dolomitasche“. Derartig morsches Gestein vermag natürlich keinen scharfkantigen Frostschutt mehr zu liefern. Andererseits verliert Frostschutt seine typische Ausbildung bei nachträglicher Einwirkung solcher Lösungsvorgänge, da denselben vor allem Ecken und Kanten ausgesetzt sind. Kleinere Gesteinskomponenten werden außerdem bei gleicher Lösungsintensität stärker angegriffen als größere. Vermorschungs- und Rundungsgrad der einzelnen Schuttkomponenten laufen somit einander ungefähr parallel. In Abb. 1 wurde dazu als Beispiel das Verhalten einer der untersuchten, eine Aussage gestattenden Fraktion $(5-20 \mathrm{~mm} \phi)$ zur Darstellung gebracht.

Das Grundprinzip der Sedimentation in der Höhle von Hunas zeigt damit unter Berücksichtigung der eingangs genannten Gesichtspunkte folgenden Ablauf vom Liegenden nach dem Hangenden:

"Sinter (und Tropfstein) - Blockschutt - Schwemmschicht - Frostschutt - Blockschutt" in einem älteren Sedimentationszyklus und: "völlig morscher, humoser Schutt Blockschutt - Frostschutt“ in einem jüngeren Zyklus, der infolge totaler Verfüllung der Höhle nicht mehr vollständig abzulaufen vermochte. Im älteren Zyklus liegt eine eingangsfernere und im jüngeren eine eingangsnahe Ausbildung vor. Beim älteren Zyklus ist außerdem die enge zeitliche Verknüpfung von Sinter mit nachfolgendem Blockschutt nicht voll gesichert.

1) Ergänzender Beitrag zu der Arbeit von Fl. Heller auf S. 111 dieses Jahrbuches. 


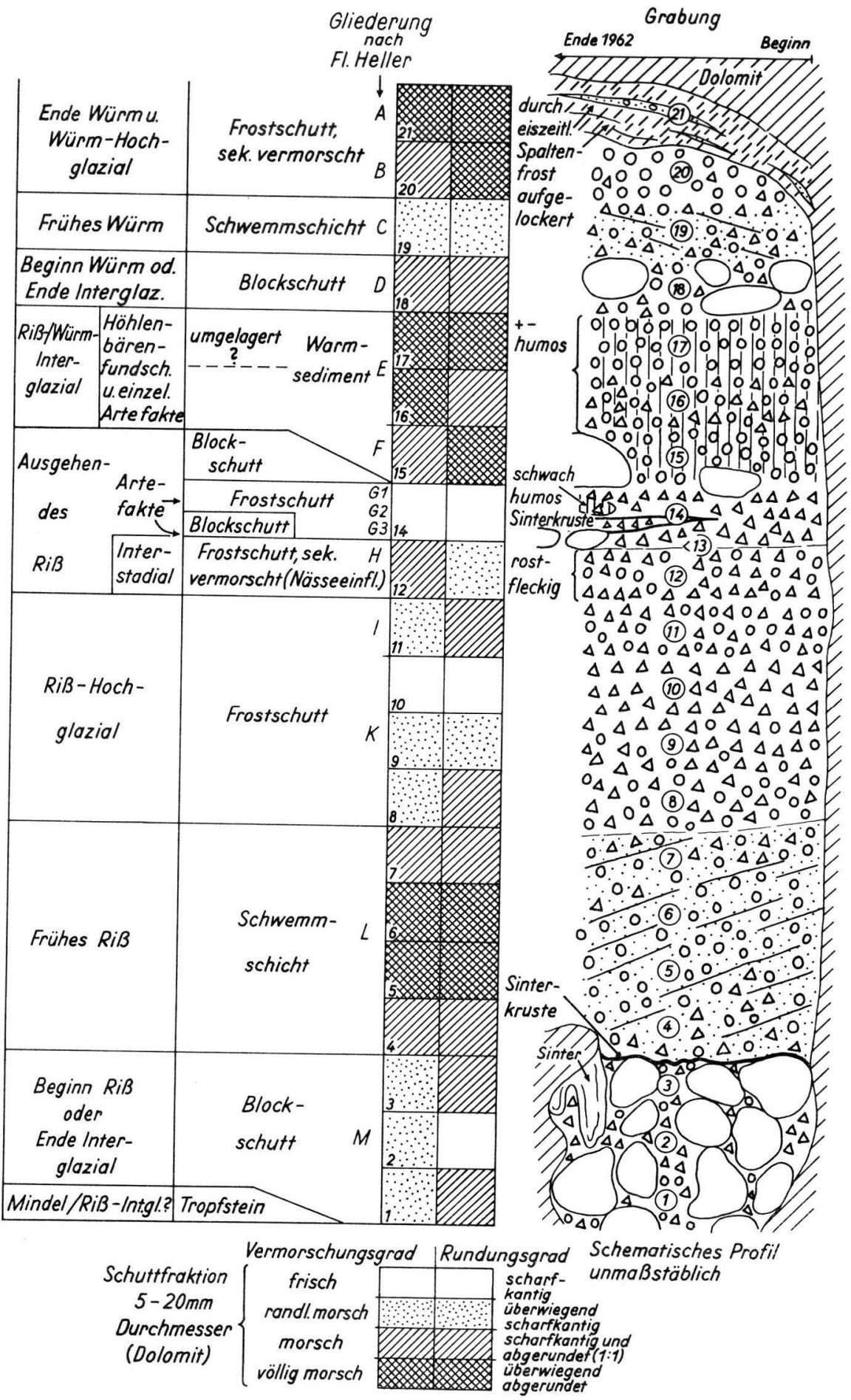

Abb. 1. Aufbau und Gliederung der Sedimente in der Höhle von Hunas (Mächtigkeit der aufgeschlossenen Verfüllung: rd. 6-7 m). 
Damit ergibt sich folgende Deutung (Abb. 1):

1. Der Sinter (älterer Zyklus?) und der humose völlig morsche Dolomitschutt (jüngerer Zyklus) gehen sicherlich auf warmklimatische Bildungsumstände zurück.

2. Der jeweils nachfolgende Blockschutt wird als Verbruch gedeutet, der durch die vorangegangene Abwitterung längs der Klüfte und Spalten am Höhlendach und die dadurch bedingte Gesteinsauflockerung ausgelöst wurde. Möglicherweise hat die in Annäherung an die nachfolgende Kaltzeit zunehmend tiefer in das Gestein greifende Frostwirkung zusätzlich mitgeholfen.

3. Die darauf folgende Schwemmschicht enthält viel Material der Oberfläche, also stark verwitterte Dolomitkomponenten (älterer Zyklus) bzw. Terra-fusca-Material (jüngerer Zyklus). Diese Schwemmschicht wird den Solifluktionsvorgängen ungefähr gleichgesetzt, die im periglazialen Raum in den Anfangsphasen einer Eiszeit besonders wirksam zu sein pflegen.

4. Darauf folgt jeweils typischer Frostschutt, der ein Ergebnis der eiszeitlichen, vorwiegend mechanischen Gesteinsaufbereitung darstellt.

5. Abschließend kommt es im älteren Zyklus noch einmal zur Bildung von Blockschutt. Entsprechend E. SCHміD (1958) wird darin eine Folge der Auflösung des Dauerfrostbodens am Ende einer Eiszeit gesehen. Im jüngeren Zyklus fehlt diese Blockschuttbildung; sie ist jedoch in einer anderen Höhle (Hohler Stein bei Schambach, südliche Frankenalb) für diesen letzteiszeitlichen Ablauf ebenfalls nachgewiesen.

Der jüngere Zyklus von Hunas umfaßt das Riß/Würm-Interglazial und die Würmeiszeit, zumindest das Frühwürm. Theoretisch wäre allerdings auch ein wesentlich höheres Alter denkbar, was aber nicht allein wegen des labilen Zustandes solcher Höhlensedimente, sondern auch aus paläontologischen und urgeschichtlichen Erwägungen unwahrscheinlich ist; denn das darunter liegende Sedimentpaket gehört damit in das Mindel/Riß-Interglazial (möglicherweise ist der Sinter noch älter?) und in die Rißeiszeit - also in stratigraphische Bereiche, aus denen bisher kaum Höhlensedimente in Mitteleuropa bekannt geworden sind.

Eine auf interstadiale Bedingungen rückgeführte Einschaltung (Vernässung mit Begleiterscheinungen) gliedert den oberen Teil des Frostschuttes der Rißeiszeit weiter auf. Begehung durch den Menschen ist hier durch Artefakte nachgewiesen. Die Grabungen haben im unmittelbar darüberliegenden, also endrißeiszeitlichen Frostschutt zahlreiche Artefakte erbracht. Bei Ende der Jahresgrabung 1962 war schließlich hier eine eingangsnahe Ausbildung aufgeschlossen, die bei der Exkursion der Deutschen Quartärvereinigung anläßlich der Tagung 1962 gezeigt werden konnte, die jedoch in Abb. 1 wegen der noch ausstehenden speziellen Untersuchung nur angedeutet ist: Dieser oberste Abschnitt der rißeiszeitlichen Ablagerungen gliedert sich jetzt auf: Frostschutt (Probe-Nr. 14) - Blockschutt humushaltiger, z. T. relativ morscher Schutt mit Artefakten - Blockschutt. Eine genauere Eingliederung dieser damit angedeuteten Unterteilung in die Detailgliederung der Rißeiszeit auf Grund der Verhältnisse im glazialen Raum bzw. der Periglazialprofile ist vorläufig nicht möglich. Doch zeigt sich dafür - und damit auch für die noch nicht einmal ältesten Begehungen durch den paläolithischen Menschen - eine Stellung im Grenzbereich des rißeiszeitlichen Frostschuttes zu dem das Riß abschließenden Blockschutt - also im Spätglazial der Rißeiszeit an.

$$
\mathrm{Sch} \text { rif t t u m (Auswahl): }
$$

Brunnacker, K.: Die Höhlensedimente im Hohlen Stein bei Schambach. - Geol. Bl. NO-Bayern, 6, S. 21-32, Erlangen 1956. 
Heller, Fl.: Ein bedeutsames Quartärprofil in einer Höhlenruine bei Hunas/Hartmannshof (Nördliche Frankenalb) 2. Vorbericht. - Eiszeitalter u. Gegenwart 13, S. 111-116, OOhringen/ Württ. 1963.

LAIs, R.: Uber Höhlensedimente. - Quartär, 3, S. 56-108, Berlin 1941.

Schmid, E.: Höhlenforschung und Sedimentanalyse - ein Beitrag zur Datierung des Alpinen Paläolithikums. - Schriften d. Inst. f. Ur- u. Frühgeschichte d. Schweiz, 13, Basel 1958.

Manuskr. eingeg. 18. 2. 1963.

Anschrift des Autors: Privatdozent Dr. Karl Brunnacker, Bayer. Geol. Landesamt, München, Prinzregentenstraße 28. 